\title{
Aging effects on sorption-desorption behaviors of PAHs in different natural organic matters
}

\author{
Zijian Wang *, Shan Chen, Yiping Xu*, Jianfeng Tang \\ State Key Laboratory of Environmental Aquatic Chemistry, Research Center for Eco-Environmental Sciences, Chinese Academy of Sciences, 18 Shuangqing Rd.,
} Haidian District, Beijing 100085, China

\section{A R T I C L E I N F O}

\section{Article history:}

Received 20 January 2012

Accepted 4 June 2012

Available online 15 June 2012

\section{Keywords:}

Aging

Dissolved humic substance

Humin

Natural organic matter

Polycyclic aromatic hydrocarbons

Sorption-desorption behavior

\begin{abstract}
A B S T R A C T
The aging effects of sorption-desorption of two polycyclic aromatic hydrocarbons (PAHs), that is, phenanthrene and chrysene on different natural organic matters (NOMs), that is, humic acid, fulvic acid, and humins of different sources were studied. Both organic carbon-normalized distribution coefficients $\left(K_{\text {oc }}\right)$ and the reversible desorption fractions $\left(f_{\text {rd }}\right)$ were evaluated. Results showed that aging effects were both sorbent and sorbate specific. With increasing aging duration, $K_{\mathrm{oc}} \mathrm{s}$ of phenanthrene on humins increased, whereas $K_{\mathrm{oc}} \mathrm{S}$ of chrysene did not change significantly. Significant $f_{\mathrm{rd}}$ declinations over aging times were observed for all the NOMs, which demonstrated the effects of aging on availability of PAHs. Furthermore, the $f_{\text {rd }}$ was not only positively correlated with the atomic $\mathrm{O} / \mathrm{C}$ ratio of NOMs, which represent the geological maturity of NOMs, but also varied with the property of sorbates. We conclude that aging effects of HOCs on NOMs should be a case-specific process and the desorption reversibility depends on the structure and characteristics of both NOMs and sorbates.
\end{abstract}

(c) 2012 Elsevier Inc. All rights reserved.

\section{Introduction}

The phenomenon that hydrophobic organic compounds (HOCs) associated with natural organic matters (NOMs) has long been reported in literatures. The sorption-desorption behavior significantly affects the bioavailability and environmental persistence of the HOCs $[1,2]$. Recently, there is growing awareness that the affinity of HOCs on NOMs depends on the composition of the NOM, solute properties, and especially the NOM-chemical contact time, which termed "aging." Aging is an important determinant of the sorption and desorption characteristics of HOCs in the environment. Previous studies of the effects of aging on mechanism-specific sorption and desorption have documented that aging time would lead to higher solute retention, reduced desorption, and less extractability with organic solvents that resulted from greater contaminant sequestration or binding [3-5]. Although some studies argued that reported sorption/desorption hysteresis phenomena may be due to experimental artifacts resulting from non-attainment of sorption equilibrium before desorption experiments, which were conceptualized as "pseudo-hysteresis" [6], true hysteresis phenomenon has been distinguished from artificial hysteresis by many researchers [7-9]. Sander et al. eliminated common artificial causes by the method of quantifying hysteresis thermodynamically and found that true sorption hysteresis was significant

\footnotetext{
* Corresponding authors. Fax: +86 1062849140.

E-mail addresses: wangzj@rcees.ac.cn (Z. Wang), ypxu@rcees.ac.cn (Y. Xu).
}

in the peat and the coal [7], and others also confirmed irreversible sorption of neutral hydrocarbons to sediments in cyclic adsorption/ desorption studies $[8,9]$. Aging appeared to have a significant impact on sorption-desorption behavior of HOCs on NOMs. For instance, it has been observed either in laboratory conditions at equilibrium or in field conditions that sorption of PAHs on soils increased with incubation time [10,11].

Although it is clear that true and certain types of artificial hysteresis has important implications for sorption-desorption behavior of organic compounds, there are limited studies on quantitative description of aging impact on availability of NOM-adsorbed organics (such as PAHs). On the other hand, although the "aging effect," reported in the polymer literature almost 40 years ago, was formerly attributed to the slow relaxation of glassy macromolecules that comprised humins [12], several unique features of the resistant desorption have been reported for the dissolved humic substance (DHS) [13]. It was also shown the solution phase concentration remained much lower than expected from equilibrium partitioning with the humic substances concentration after aging $[3,14]$. However, most attention focused on the sorption by DHS in single-step uptake experiments while little has been paid to the reversibility of sorption by including desorption steps. Consequently, the enhanced retention was usually neglected when processing binding data of HOC-DHS interactions according to the partitioning concept and application of this inaccurate view can greatly affect attempts to model the transport and fate of organic pollutants in natural systems [15]. Since little experiment has been 
conducted on the influence of aging on chemicals bound with DHS, it is necessary to provide an improved understanding of the relationship between chemical retardation in NOMs and aging time.

In order to capture the hysteresis of desorption isotherms, numerical models that incorporate NOM heterogeneity have been proposed. Weber et al. introduced a composite model, the distributed reactivity model for sorption by soils and sediments to characterize intrinsic heterogeneities in the properties and behaviors of soils and sediments [16]. A similar model was applied by Schlebaum in a study with humic acid (HA) [17]. These models were parallel models with discrete sites that may have different affinities for HOCs and different sorption mechanisms. Another kind of model often used is the two-compartment model with two sites in series [18]. It is assumed that the two compartments differ only in kinetics and not in their affinity for the HOCs. Desorption was characterized by two types of processes: readily desorption fraction, and a fraction that does not desorb over experimental time scales. All those models required to derive case-by-case parameters to reduce the uncertainty.

In our study, a two-site desorption model based on passive sampling approach was proposed to investigate the effect of NOM-chemical aging time on sorption and desorption of phenanthrene and chrysene. Our previous work has shown that the uptake rate of HOCs in triolein-embedded cellulose acetate membrane (TECAM) is independent on HA concentrations [19]. Thereby it can be convinced that the presence of NOM does not affect the uptake kinetics by TECAM, so it was used to quantitate the free concentration of analyte in NOM-containing samples and acting as a sink to sequestrate freely dissolved solute [20].

To understand structures of NOMs, elemental and nuclear magnetic resonance data were used. Comparison was made between humin and DHS with respect to association affinity, desorption resistant fraction, as well as variation of these parameters related to aging time, and physiochemical properties of NOM accordingly. Macroscopic observations are used to make inferences about sorption mechanisms and the physicochemical factors affecting the desorption resistance of PAHs by NOM.

\section{Materials and methods}

\subsection{Reagents and chemicals}

Two deuterated PAH standards phenanthrene- $d_{10}$ and chrysene- $d_{12}$ were purchased from Supelco Inc. (Bellefonte, PA). The mixed standard solution was prepared by diluting the original stock solutions with methanol to $20 \mathrm{mg} / \mathrm{L}$. Five NOM samples obtained from various sources were used in the present study, including two kinds of standard humic and fulvic acids, Nordic aquatic fulvic acid reference (Nordic FA) and Leonardite HA standard (International Humic Substances Society, Denver, USA), as well as a kind of natural humic acid isolated from pond sediment (PSHA), and two kinds of humins isolated from pond sediment, PSBC (black carbon) and PSKB (kerogen + black carbon). All the NOMs from pond sediment were generous gifts provided by Prof. Ping-An PENG, Guangzhou Institute of Geochemistry, Chinese Academy of Sciences. Stock solutions of dissolved FA, Leonardite HA, and PSHA were prepared as described previously [21]. All NOMs were sterilized using a conventional multiple autoclaving procedure. The organic carbon content of three DHS stock solutions was measured with a total organic carbon analyzer (phoenix-8000, Tekmar-Dohrmann, Mason, OH). DHS sample solutions were produced through diluting the stock solution with ultrapure water to reach content of $5 \mathrm{mg} \mathrm{C/L}$. The solution was then adjusted to $\mathrm{pH} 7.0 \pm 0.3$ with $0.1 \mathrm{M} \mathrm{HCl}$ and $0.1 \mathrm{M} \mathrm{NaOH}$. PSKB and PSBC suspensions were produced individually by weighing corresponding weights to reach content of $5 \mathrm{mg} \mathrm{C} / \mathrm{L}$ and then were dispersed by sonic oscillation. All sample solutions were transferred to $1 \mathrm{~L}$ sterilized glass flasks and spiked with $500 \mathrm{ng} / \mathrm{L}$ PAHs. $\mathrm{HgCl}_{2}$ $(50 \mathrm{mg} / \mathrm{L})$ was added to prevent microbial degradation. The ultrapure water $(\mathrm{OC}<0.1 \mathrm{mg} \mathrm{C} / \mathrm{L})$ used in this study was purified by a Milli-Q Gradient system (Millipore, Bedford, MA). Solvent and other chemicals used in the study were of GC or analytical reagent grade. Small pieces of TECAM $(4 \mathrm{~cm} \times 6 \mathrm{~cm})$ were used and the procedure for preparing was the same as described previously [22].

\subsection{Sorption and desorption experiments}

The distribution coefficients of phenanthrene and chrysene on NOMs were determined in sorption experiments. The uptake profiles of PAHs in TECAM were produced in NOM-free solutions. It was observed that when immersed in PAHs solutions in the absence of NOM and shaken for $1 \mathrm{~h}$, TECAM accumulated less than $5 \%$ of total amount PAHs, suggesting that the depletion of TECAM was negligible in $1 \mathrm{~h}$ sampling. Uptake-time plots of TECAM in $100 \mathrm{ng} / \mathrm{L}$ PAHs solutions over $12 \mathrm{~h}$ showed that accumulation is in the linear-uptake phase when test solutions were changed per hour (Fig. S1 in Supplementary material). Therefore, the slopes of uptake profiles of PAHs in TECAM over $12 \mathrm{~h}$ were produced in a series of water samples (spiked with 20-1000 ng/L PAHs, the concentration of carrier did not exceed $0.1 \mathrm{~mL} / \mathrm{L}$ ) to calculate intrinsic uptake rate $k_{1}$ according to the following equation:

$C_{m}=k_{1} C_{w} t$

where $C_{m}$ is the concentration of target compound in the TECAMs; $k_{1}$ represents the true rate constant of mass transfer from water phase to TECAMs; $C_{w}$ is the free analyte concentration; and $t$ is the sampling time. Subsequent experiments were carried out in the presence of NOM for aging times ranging from 2 to 32 days. $5 \mathrm{mg} \mathrm{C} / \mathrm{L}$ NOM was placed in flasks and contacted with $1 \mathrm{~L}$ of PAHs solution. The flasks were kept sealed with Teflon-lined caps, shaken gently on the shaker in the dark at $20 \pm 1{ }^{\circ} \mathrm{C}$ during the whole aging period. Then, the apparent uptake rate $k_{1}^{\prime}$ was determined as follows:

$C_{m}=k_{1}^{\prime} C_{t} t$

where $k_{1}^{\prime}$ represents the apparent uptake rate of TECAM when NOM is present; $C_{t}$ is the total concentration of analyte. Control experiments of NOM-free solutions in triplicate run simultaneously to measure the loss of PAHs during aging time (results can be referred in Supplementary material). PAH concentrations after accumulation were measured by liquid-liquid extraction. Mass balances were conducted by carefully recovering the solute and the retrieved concentrations after aging were used as total amount of PAHs in all the calculation.

The relationship between organic carbon-normalized partition coefficient $\left(K_{\mathrm{oc}}\right)$ for NOM and target concentrations in NOM can be expressed by the Freundlich isotherm model [16]:

$C_{\text {oc }}=K_{\mathrm{oc}} C_{W}^{n}$

where $C_{\mathrm{oc}}$ and $C_{w}$ are the sorbent-phase and aqueous-phase solute free concentrations; $n$ is the nonlinearity index of the sorption process. Although some researchers had reported that high levels of complex matrixes enhanced the diffusive mass transfer through the unstirred boundary layer [23], our previous study has shown that the uptake of TECAM is HA-independent in relatively low HA content of $15 \mathrm{mg} \mathrm{C/L} \mathrm{[19].} \mathrm{Since} \mathrm{the} \mathrm{true} \mathrm{uptake} \mathrm{rate} \mathrm{of} \mathrm{HOCs} \mathrm{in} \mathrm{TE-}$ CAM is not influenced by the presence of NOM, $n$ and $K_{\text {oc }}$ can be determined from the equation as follows:

$\log \left(\frac{k_{1}}{k_{1}^{\prime}}-1\right)=\log K_{\mathrm{oc}}+(n-1) \log C_{w}[\mathrm{OC}]$ 
where $[\mathrm{OC}]$ represents the organic carbon-normalized concentration of NOM, and aqueous concentrations were calculated according to Eq. (1) using obtained $k_{1}$.

Desorption experiments were conducted with a laboratory static aqueous method. The $500 \mathrm{ng} / \mathrm{L}$ PAH solution $(1 \mathrm{~L})$ after aging was placed into a conical flask and deployed with four pieces of TECAM for $24 \mathrm{~h}$. To prevent losses of PAHs due to volatilization, the flask was sealed and shaken on a constant temperature-reciprocating shaker throughout the whole accumulation course. Temperature was kept at $20 \pm 1{ }^{\circ} \mathrm{C}$ in all experiments. After TECAMs are introduced into a NOM-water system, desorption occurred from NOM to water. In condition that part of the bound compound does not take part in partition between water phase and NOM phase, or this fraction is desorption resistant during the exposure period, a mass balance of solute can be expressed by the following equation:

$C_{m}=\left(C_{w 0}+C_{w 0}^{n} K_{o c}[O C] f_{r d}-C_{w 1}^{n} K_{o c}[O C] f_{r d}-C_{w 1}\right) / M$

where $f_{\text {rd }}$ represents the reversible desorption fraction in the presence of NOM; $C_{w 0}$ and $C_{w 1}$ are the initial aqueous concentration and the final aqueous concentration of analyte before and after TECAMs were introduced. All the experiments were carried out in triplicate.

Analytical processing was performed as previously described [21]. Surrogate standard naphthalene- $d_{8}$ was added for each batch and PAH concentrations in TECAM were corrected by the recovery of blank spikes. Instrumental analysis was detailed in Supplementary material.

\section{Results and discussion}

\subsection{Sorption isotherms}

Origin 8 (Microcal Software, Northampton, MA) was used to fit all the data by linear regression. Typical Freundlich adsorption profiles can be found in Supplementary material (Fig. 1). As there was no significant difference in " $n$ " for different aging times $(p>0.05)$, only the $n$ values measured after 2-day aging were used in the following study. Sorption isotherms in DHS were almost linear as the values of $n$ ranged from 0.97 to 1.06 . This result was in consistent with the study of Pan et al. where the isotherm nonlinearity factors of PAHs in DHS were approached to 1 closely when consolute (another PAH) was present [13]. To simplify the calculation, the $n$ values of DHS were all set as 1 in the following study. The $n$ values of PSKB and PSBC were very close to each other for the same PAH ( 0.69 and 0.68 for phenanthrene and 0.84 and 0.83 for chrysene, respectively), but increases as the $K_{\mathrm{OW}}$ of PAHs increase. Similar

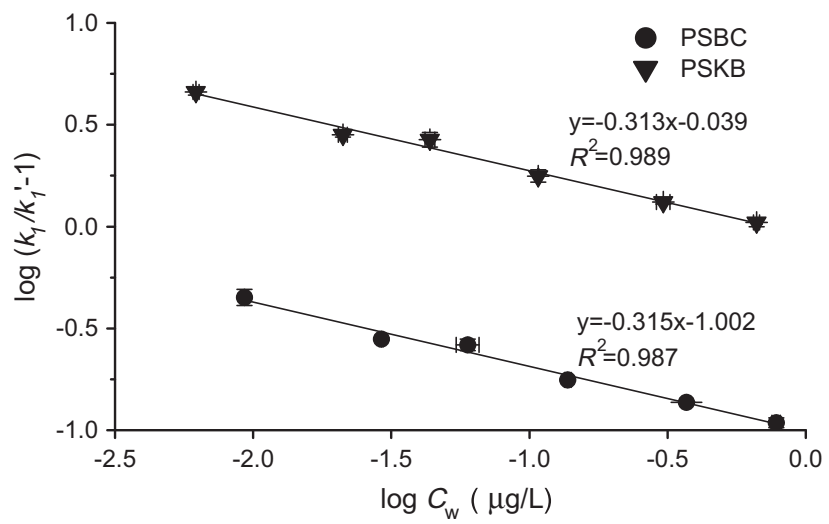

Fig. 1. Sorption isotherms of phenanthrene in PSBC (black carbon isolated from pond sediment) and PSKB (kerogen and black carbon isolated from pond sediment) after 2-day aging. Plots represent the mean $(n=3$ replicates $) \pm$ standard deviations phenomenon has been observed by Bucheli that a higher degree of nonlinearity (smaller " $n$ ") for more hydrophobic PAH in sootwater distribution [24]. According to their explanation, sorption in humin was more concentration-dependent for chrysene- $d_{12}$ because its larger surface area ( $188 \AA^{2}$ compared to phenanthrene$d_{10}$ of $152 \AA^{2}$, calculated by ChemOffice) yields higher surface coverage.

Single-point $K_{\mathrm{oc}}$ values at $C_{w}=100 \mathrm{ng} / \mathrm{L}$ were also calculated for comparison with DHS. Corresponding results for all NOM summarized in Table 1 reflected difference in sorption kinetics of different PAHs in different NOM. The strongest sorption occurred in PSKB after aging, about 20 times higher for phenanthrene and five times higher for chrysene than the weakest sorption in Nordic FA. It is as expected that individual NOM has stronger sorption for chrysene than phenanthrene. Generally, $K_{\mathrm{oc}}$ values are markedly higher after aging for 32 days than 2 days (by a factor of 2-9) for phenanthrene in humins, with slight increase for both PAHs in PSHA. The significant influence of aging on sorption of phenanthrene illustrated the existence of slow sorption in humins.

It was not clear why there was no obvious change for chrysene sorption in all NOMs. Plausible explanation might be the limited accessibility to microporous domains of organic matrices for the larger molecular volume of chrysene than phenanthrene (447 and $367 \AA^{3}$, respectively) [25]. In an earlier study, lower adsorbed mass of pyrene compared to phenanthrene for the same sorbent was observed and it was supposed that structural rearrangement of the soil organic matrix in addition to slow diffusion into microporous domains were more pronounced with the adsorption of phenanthrene after sample aging [26]. Similar to our results, Ball and Roberts also attributed the very low sorption rates of HOCs in aquifer material to strong retardation and hindrance of transport within the microporous solids [27]. It is possible that the inner sorption site of NOM might not be easily accessible for chrysene because they were blocked by other organic material.

Relationships between aromatic content and sorption parameters were usually expected [2]. Nevertheless in this work, the correlation of $K_{\text {oc }}$ with NOM properties such as $\mathrm{H} / \mathrm{C}, \mathrm{O} / \mathrm{C}$ as well as NOM aromaticity were all unapparent for both PAHs $(p>0.1)$ (properties of NOMs were listed in Table S3 in Supplementary material). These phenomena may mainly due to the source and structural difference between DHS and humins. As usually classified, HA and FA are typed into amorphous humic substances which are loosely packed, while humins pose rigid geosorbent characteristics [15]. And this may reflect that the use of only macroscopic properties is unlikely to underlie the sorptive character of a geosorbent. It was also noteworthy that sorption of chrysene in PSBC was weaker than Leonardite HA and PSHA. Theoretically, the high microporosity of $\mathrm{BC}$ allows for a proportionately higher surface area than humic materials. Nonetheless, BC is less reactive than sediment NOMs and speculated to be more condensed even than diagenetically matured kerogen [2]. It is known that kerogen and $\mathrm{BC}$ matrices have backbone structures of aromatic molecule stacks and the interlayer spacing decreases as a function of condensation or maturation. Petrographic analysis results of PSBC showed spheroidal shapes with smooth and homogeneous surfaces, vitrinite, and semifusinite macerals [28], reflecting that PSBC was highly matured and condensed. It was also found that specific surface area of PSBC $\left(1 \mathrm{~m}^{2} / \mathrm{g}\right)$ was only half of the PSKB $\left(2 \mathrm{~m}^{2} / \mathrm{g}\right)$. What is more, internal microvoids in geological sorbent were accessible only by diffusion through the solid phase and the diffusion was controlled by steric hindrance within pore spaces. In fact, Popovicheva et al. reported that the size of micropores in combustor-generated soot was in the range of 1.1-1.7 nm, and kerosene soot exhibits ultramicroporosity with the size of $0.52 \mathrm{~nm}$ and a smaller micropore volume [29]. Since the wide of chrysene and phenanthrene was 13.9 and $11.8 \AA$, respectively, [25], we speculated that 
Table 1

Single-point $K_{\text {oc }}$ values $\left(\times 10^{3} \mathrm{~L} / \mathrm{kg} \mathrm{C}\right)$ of five NOMs at $C_{w}=100 \mathrm{ng} / \mathrm{L}$.

\begin{tabular}{|c|c|c|c|c|c|c|}
\hline \multirow[t]{2}{*}{ Sample } & \multicolumn{3}{|c|}{$K_{\text {oc }}$ of phenanthrene } & \multicolumn{3}{|c|}{$K_{\text {oc }}$ of chrysene } \\
\hline & $2 \mathrm{~d}$ & $16 \mathrm{~d}$ & $32 \mathrm{~d}$ & $2 \mathrm{~d}$ & $16 \mathrm{~d}$ & $32 \mathrm{~d}$ \\
\hline Nordic FA ${ }^{b}$ & $30(1)^{a}$ & $31(2)$ & $34(2)$ & 428 (49) & $446(21)$ & $422(8)$ \\
\hline Leonardite $\mathrm{HA}^{\mathrm{b}}$ & $75(5)$ & $62(4)$ & $71(4)$ & $911(89)$ & $1083(26)$ & $993(35)$ \\
\hline PSHA $^{\mathrm{b}}$ & $52(4)$ & $61(4)$ & $82(12)$ & 1534 (133) & $1698(99)$ & 1824 (183) \\
\hline PSKB $^{\mathrm{b}}$ & $376(5)$ & $405(5)$ & $752(46)$ & $2096(64)$ & 1946 (208) & 1909 (142) \\
\hline $\mathrm{PSBC}^{\mathrm{b}}$ & $41(2)$ & $101(3)$ & $371(24)$ & $675(47)$ & $697(68)$ & $687(31)$ \\
\hline
\end{tabular}

a Values in parentheses represent standard deviations

b (Nordic FA) Nordic aquatic fulvic acid reference; (Leonardite HA) Leonardite humic acid standard; (PSHA) humic acid isolated from pond sediment; (PSBC) black carbon isolated from pond sediment; (PSKB) kerogen and black carbon isolated from pond sediment.

the similar PSBC was too condensed for absorption or penetration of chrysene into internal microvoids, while phenanthrene was sterically hindered than chrysene in PSBC because of its smaller molecular volume, and could be allowed to access inner sorption regions at a relatively faster rate.

\subsection{Effects of aging and chemical property on desorption}

Artificial desorption resistant could be a manifestation resulting from increasing distribution coefficients after desorption was started. However, in such a short contact time of $24 \mathrm{~h}$, underestimation of $f_{\text {rd }}$ can be diminished to an unnoticeable degree. Results showed that for both PAHs in all NOM used in this study, $f_{\text {rd }}$ declined over aging times (Fig. 2), illustrating the effects of aging on availability of PAHs. These results agree with an earlier work which found that the availability of PAHs decreased with length
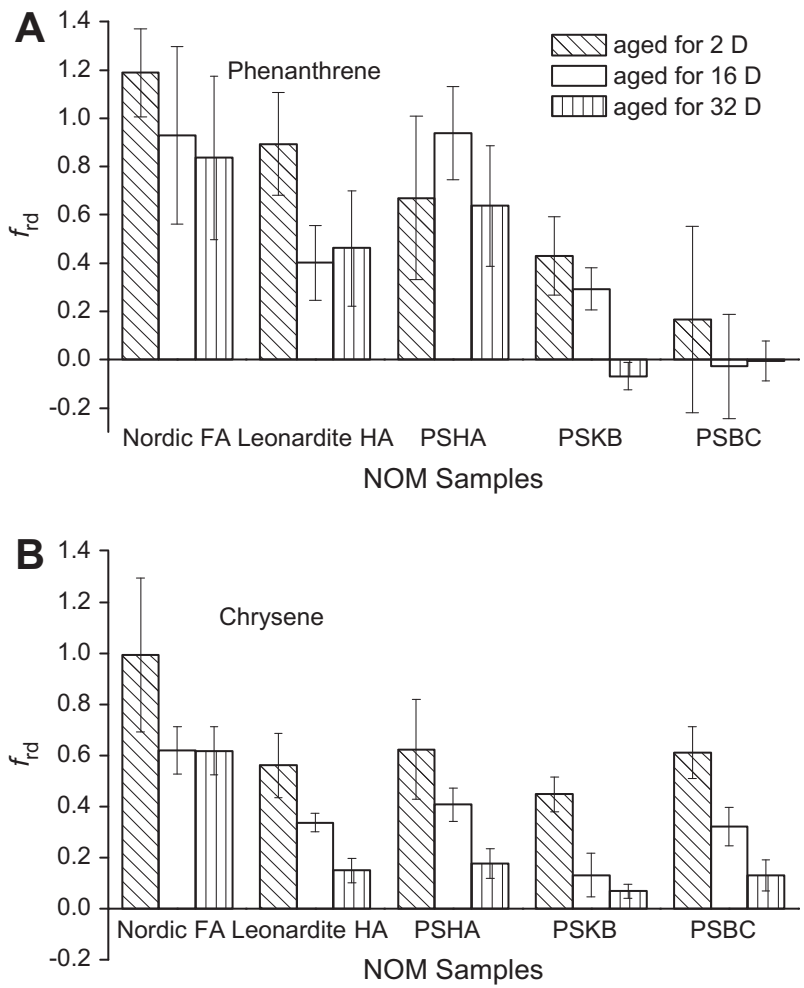

Fig. 2. Reversible desorption fraction $\left(f_{\text {rd }}\right)$ of two PAHs: (A) phenanthrene and (B) chrysene for different aging times in various NOMs used in this study: (Nordic FA) Nordic aquatic fulvic acid reference; (Leonardite HA) Leonardite humic acid standard; (PSHA) humic acid isolated from pond sediment; (PSBC) black carbon isolated from pond sediment; (PSKB) kerogen and black carbon isolated from pond sediment. The error bars represent the standard deviations calculated according to error propagation. of aging in sterile soil, sediment, and dissolved organic matter (DOM) [30-32]. In those studies, the time scale was comparable to that used in the present study. And the increase in slow desorption fraction of PAHs was explained by the proceeding diffusion into the slowly exchanging part and by the presence of the solute at more remote locations from which desorption is slower [31].

Lower hydrophobic phenanthrene seemed to desorb more easily from DHS. This is probably reasonable as stronger molecular polarizability of chrysene would increase the strength of the van der Waals attraction forces and thereby the sorption affinity, while phenanthrene may be more weakly associated with this structural component and was easily desorbed during TECAM accumulation [2]. Although our sorption experiment showed that 32 days may not sufficiently long for phenanthrene to obtain apparent sorption equilibrium in humin prior to initiation of desorption in this study, some previous studies have shown that aging beyond 3 weeks to several months has negligible additional effect on desorption resistant fractions or release rates [33]. It appeared that humin-sorbed PAHs were more recalcitrant than those in DHS after aging. For PSBC and PSKB particularly, desorption resistant fractions increased as a function of aging by factors of $2-10$, while sorption irreversibility did not increase significantly with aging in Nordic FA. It was found that $f_{\text {rd }}$ of two PAHs had a strong positive linear relationship to $\mathrm{O} / \mathrm{C}$ atomic ratio of NOM after different aging periods $(p<0.05)$ and the adjusted coefficient of determination $\left(R^{2}\right)$ of correlations ranged from 0.68 to 0.91 (Fig. 3). Nevertheless, no correlation could be observed between the $f_{\text {rd }}$ and NOM aromaticity $(p>0.1)$ (aromaticity parameters of NOMs were listed in Table S3 in Supplementary material). The result is consistent with the observation of Huang et al. that hysteretic behaviors of phenanthrene in geosorbents correlate inversely with the $\mathrm{O} / \mathrm{C}$ atomic ratio and indicated that condensed samples exhibited greater solute affinity, and more pronounced hysteresis [34]. As the O/C atomic ratio of soil organic matter decreased with diagenetic alteration [16], it may represent important factor of geosorbent maturity. In other words, PAHs seemed to be more recalcitrant in diagenetically mature geosorbents.

Solute properties were also found to have important influence on desorption. We hypothesized that some of the retardation could have resulted from steric effects. Brusseau et al. claimed the size of the "pores" associated with organic matter is similar to the geometrical parameters of the sorbate molecules [35]. The lower desorption reversibility of chrysene than phenanthrene in DHS may also serve as the evidence to support our suspicion of steric hindrance influence on desorption.

\subsection{Possible mechanisms contributing to desorption resistance}

Our results showed that although aging had little effect on sorption of chrysene in humins, it significantly increased the desorption resistance. This discrepancy may illustrate that aging influences desorption in different mechanisms from sorption. To 

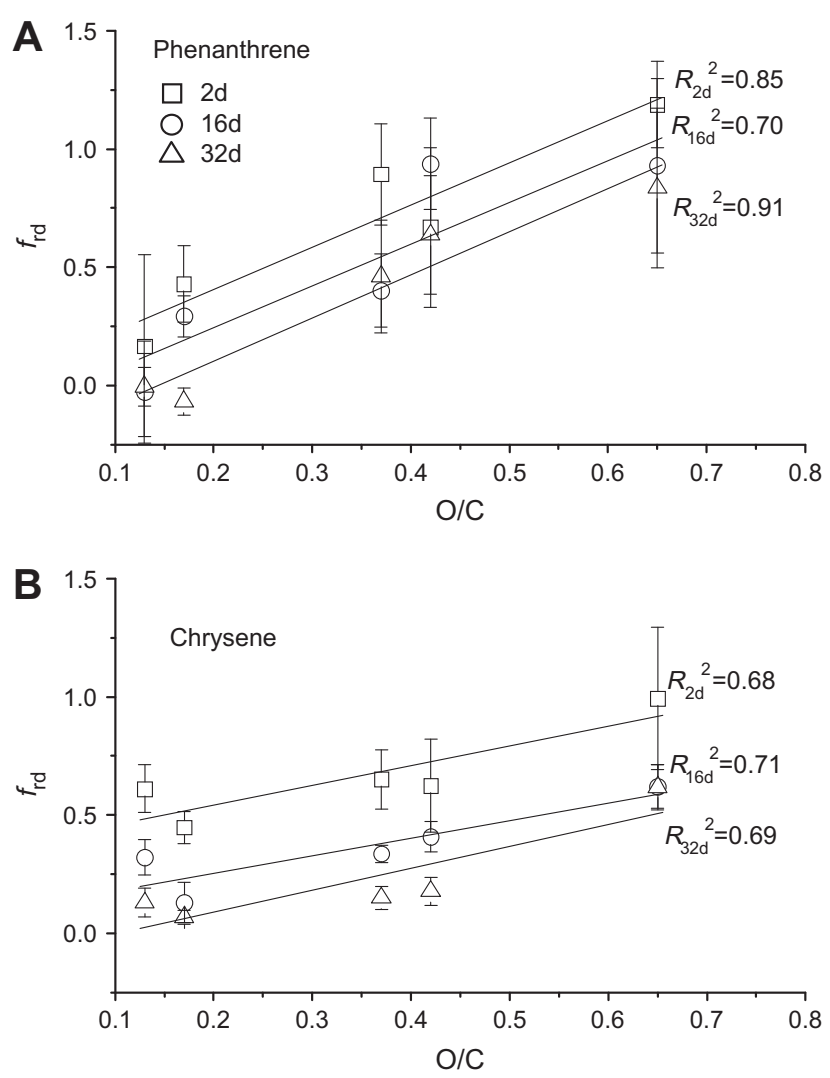

Fig. 3. Correlations between reversible desorption fraction $\left(f_{\text {rd }}\right)$ of two PAHs: (A) phenanthrene and (B) chrysene and $\mathrm{O} / \mathrm{C}$ atomic ratios of NOMs after different aging times. The error bars represent the standard deviations calculated according to error propagation, and the values of $R^{2}$ represent the adjusted coefficients of determination.

account for the observations of the desorption resistance, several hypotheses have been developed in last decades. Most prevalent theories therein include physical entrapment within micro- and nanopores, as well as irreversible pore deformation. It was declared that formation of meta-stable states of adsorbate in fixed mesopores on external surfaces or in the internal voids of soil organic matter was largely responsible for the great entrapment of PAHs in humin [7]. However, it was also suggested that pore deformation plays a major role to the observed hysteresis in kerogen compared to physical entrapment of sorbate molecules [36]. Direct confirmation of the "porous" nature of organic matter has been reported. The internal microstructure of NOM responds to changes in environmental stress such as sorbate concentration. As the sorbate becomes diluted, segmental flexibility of solid decreases. These processes are only partly reversible and sorbate molecules are entrapped in the sorbent consequently $[12,35]$. It was found that physical reorientation of sorption domains can produce contacttime-dependent changes in the phase-distribution behaviors of organic solutes in soil organic matter [3].

Our results also showed that a small fraction of solute, upon sorption, is trapped unexpectedly in PSHA and Leonardite HA, especially after aging. Two broad explanations are possible. First, the enhanced desorption resistance of PAHs in DHS may merit alternative mechanistic interpretations other than partitioning concept because of the structural heterogeneity of HA. Hu et al. found that crystalline polyethylene-like domains existed in HA and proposed that the presence of the poly(methylene)crystallites may contribute specific properties [37]. Dissolved HA has been proved to contain semimicelle or pseudomicelle structures even below the critical micelle concentration [38]. When several dissolved HA molecules come together to form bigger aggregates, a micelle-like conformation could be formed because of the cross-linking of dissolved HA molecules [13]. Exchanging in either constricted microporous channels or glassy/crystalline organic phase in the interior compartment is usually expected to be extremely slow [31]. Therefore, although unapparently, the enhancement of PAH sorption in PSHA provided a possibility that slow desorption take place after aging because of the presence of micelle-like regions. Simultaneously, sorption sites themselves may undergo conformational changes, bringing in sequestration of the solute in hydrophobic micelle cage regions of the HA [39]. The second possible cause of desorption resistance is that after aging, the structural realignment of the flexible, cross-linked network could cause different microscopic pathways for sorptive uptake and release process, which result in desorption resistance. The latter possibility appears more effective as compared to similar results reported by a number of studies. In Schlebaum et al.'s study, results showed that part of pentachlorobenzene sorbed to a dissolved humic acid resisted desorption. And they proposed the likely mechanism that the non-labile fraction was a consequence of a change of conformation of the HA after the binding of pentachlorobenzene [17]. Ge et al. observed a more compact conformation of humic substance assemblies after the sorption of naphthalene using atomic force microscopy [40]. As a result, it is possible that the conformation of DOM was changed after binding with solute, which resulted in resistant desorption.

\section{Conclusions}

In summary, we have shown the varied sorption-desorption behaviors of two PAHs in different DHSs and humins after aging. The significant influence of aging on sorption was only observed for phenanthrene, and the most plausible explanation was that larger molecular volume of chrysene than phenanthrene limited its accessibility to microporous domains of NOMs. The availability of PAHs estimated as the reversible desorption fraction $\left(f_{\text {rd }}\right)$ declined over aging times for all the NOMs. However, the humin-sorbed PAHs were more recalcitrant than those in DHS after aging, and the $f_{\text {rd }}$ of PAHs had a strong positive linear relationship to $\mathrm{O} / \mathrm{C}$ atomic ratios of NOMs, which may represent important factor of geosorbent maturity. In addition, desorption resistance was also found influenced by solute properties which may be owing to the molecular volume. As mentioned above, the aging effect on mobility and availability of NOM-associated HOCs should be examined differently, as it was a kind of complicated process affected by the structure and characteristics of both the NOMs and sorbates.

\section{Acknowledgments}

This study was financially supported by the Chinese Academy of Science (KZCX2-YW-Q02-06), National Natural Science Foundation of China (40801204, 20921063), and State Key Laboratory of Environmental Aquatic Chemistry (10Y03ESPCR). We particularly appreciate the generosity of Prof. Ping-An PENG and Dr. Jian-Zhong SONG (Guangzhou Institute of Geochemistry, Chinese Academy of Sciences, China) for providing PSBC and PSKB samples.

\section{Appendix A. Supplementary material}

Supplementary data associated with this article can be found, in the online version, at http://dx.doi.org/10.1016/j.jcis.2012.06.003.

\section{References}

[1] X. Louchart, M. Voltz, Environ. Sci. Technol. 41 (2007) 1137.

[2] Cm. Golding, R.J. Smernik, G.F. Birch, Environ. Sci. Technol. 39 (2005) 3925.

[3] M. Palomo, A. Bhandari, Environ. Sci. Technol. 40 (2006) 3402. 
[4] H.M. Lesan, A. Bhandari, Water Res. 37 (2003) 1644

[5] J.P. Gao, J. Maguhn, P. Spitzauer, A. Kettrup, Water Res. 32 (1998) 2089.

[6] G. Wang, S. Kleineidam, P. Grathwohl, Environ. Sci. Technol. 41 (2007) 1186

[7] M. Sander, Y. Lu, J.J.J. Pignatello, Environ. Qual. 34 (2005) 1063.

[8] A.T. Kan, G. Fu, M.A. Hunter, M.B. Tomson, Environ. Sci. Technol. 31 (1997) 2176.

[9] W. Chen, A.T. Kan, M.B. Tomson, Environ. Sci. Technol. 34 (2000) 385.

[10] V.J. Bierman, Environ. Sci. Technol. 24 (1990) 1407.

[11] D.P. Weston, L.M. Mayer, Environ. Toxicol. Chem. 17 (1998) 830.

[12] M. Sander, Y. Lu, J.J. Pignatello, Environ. Sci. Technol. 40 (2006) 170.

[13] B. Pan, S. Ghosh, B. Xing, Environ. Sci. Technol. 41 (2007) 6472.

[14] M. Haitzer, B.K. Burnison, S. Höss, W. Traunspurger, C.E.W. Steinberg, Environ. Toxicol. Chem. 18 (1999) 459.

[15] G. Cornelissen, G.D. Breedveld, S. Kalaitzidis, K. Christanis, A. Kibsgaard, A.M.P. Oen, Environ. Sci. Technol. 40 (2006) 1197.

[16] W.J. Weber, P.M. McGinley, L.E. Katz, Environ. Sci. Technol. 26 (1992) 1955

[17] W. Schlebaum, A. Badora, G. Schraa, W. van Riemsdijk, Environ. Sci. Technol. 32 (1998) 2273.

[18] J.J. Pignatello, F.J. Ferrandino, L.Q. Huang, Environ. Sci. Technol. 27 (1993) 1563.

[19] R. Ke, Z. Wang, S. Huang, S.U. Khan, Anal. Bioanal. Chem. 387 (2007) 2871.

[20] S. Chen, Y. Xu, Z. Wang, J. Colloid Interf. Sci. 350 (2010) 348.

[21] S. Chen, R. Ke, S. Huang, L. Sun, J. Zha, Z. Wang, Chinese Sci. Bull. 52 (2007) 2642.
[22] Y. Xu, Z. Wang, R. Ke, S.U. Khan, Environ. Sci. Technol. 39 (2005) 1152.

[23] A.G. Oomen, P. Mayer, J. Tolls, Anal. Chem. 72 (2000) 2802.

[24] T.D. Bucheli, O. Gustafsson, Environ. Sci. Technol. 34 (2000) 5144.

[25] M.M.C. Ferreira, Chemosphere 44 (2001) 125.

26] A. Abu, S. Smith, Environ. Sci. Technol. 39 (2005) 7585.

[27] W.P. Ball, P.V. Roberts, Environ. Sci. Technol. 25 (1991) 1223.

[28] J. Song, P. Peng, W. Huang, Environ. Sci. Technol. 36 (2002) 3960.

29] O.B. Popovicheva, N.M. Persiantseva, B.V. Kuznetsov, T.A. Rakhmanova, N.K. Shonija, J. Suzanne, D.J. Ferry, J. Phys. Chem. A. 107 (2003) 10046.

[30] P.B. Hatzinger, M. Alexander, Environ. Sci. Technol. 29 (1995) 537.

[31] G. Cornelissen, P.C.M. van Noort, H.A.J. Govers, Environ. Toxicol. Chem. 16 (1997) 1351.

[32] S. Johnson, Sci. Total Environ. 67 (1987) 269.

[33] M.D. Johnson, W. Huang, W.J. Weber, Environ. Sci. Technol. 35 (2001) 1680.

[34] W. Huang, W.J. Weber, Environ. Sci. Technol. 31 (1997) 2562.

[35] M.L. Brusseau, R.E. Jessup, P.S.C. Rao, Environ. Sci. Technol. 25 (1991) 134.

[36] M. Sander, J.J. Pignatello, Environ. Sci. Technol. 39 (2005) 7476.

[37] W.G. Hu, J. Mao, B. Xing, K. Schmidt-Rohr, Environ. Sci. Technol. 34 (2000) 530

38] R.L. Wershaw, Environ. Sci. Technol. 27 (1993) 814.

[39] W.J. Braida, J.J. Pignatello, Y. Lu, P.I. Ravikovitch, A.V. Neimark, B. Xing, Environ. Sci. Technol. 37 (2003) 409.

[40] X.P. Ge, Y.M. Zhou, C.H. Lu, H.X. Tang, Sci. China Ser. B: Chem. 49 (2006) 256 